\title{
Mind the trap
}

\author{
Trapping objects using light is a well-known technique. But designing traps that are \\ subwavelength in size is a less well-explored avenue. Nature Photonics spoke to \\ Alexander Grigorenko about the potential benefits.
}

\begin{abstract}
Your work is about nanometric optical tweezers. What are they?

Nanometric optical tweezers are optical traps with trapping volumes much smaller than the wavelength of the laser light used to create the trap. Conventional optical tweezers use ordinary (far-field) photons to trap objects, and the size of the trap is on the scale of the wavelength. But in a nanometric trap, we rely on virtual photons and their near-fields to trap objects in subwavelength volumes. Virtual photons can be packed into a much tighter space (say, $10 \mathrm{~nm}$ instead of $500 \mathrm{~nm}$ ) and can apply much stronger forces to objects. As a result, nanotweezers offer much stronger traps than conventional tweezers for the same laser intensity.

You can actually perform nanometric trapping using ordinary laser tweezers if you ramp up the laser power to a high enough level. But the advantage of nanoscale traps is that the same trapping efficiency can be achieved with a lot less power, which is therefore more promising for trapping fragile (biological) objects without destroying or damaging them. That is the whole idea behind this work.
\end{abstract}

So what comprises your nanoscale traps? We use pairs of gold nanopillars fabricated on a glass substrate to form very well-defined, three-dimensional plasmonic traps. The pillars are about $100 \mathrm{~nm}$ to $140 \mathrm{~nm}$ in diameter, and are separated typically by $140 \mathrm{~nm}$ from other pairs. The important parameter is the gap between the two pillars, which, using nanolithography, can be reduced down to as little as $10 \mathrm{~nm}$. Roughly speaking, the gap determines the actual size of the trap and defines the maximum impulse that can be transferred to a trapped object (the impulse is inversely proportional to the gap size).

Why nanopillars?

People have considered using nano-sized needles to perform optical tweezing. But to create a proper three-dimensional trap, the needle has to be very, very sharp, with a radius of curvature of say $5 \mathrm{~nm}-$ much sharper than current nanolithography

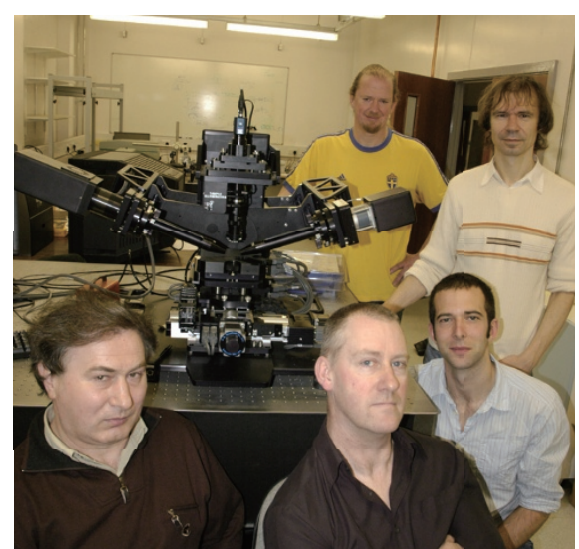

Optical trappists. From left to right, sitting: Vasyl Kravets, Mark Dickinson, Nicholas Roberts. From left to right, standing: Fredrik Schedin, Alexander Grigorenko.

can provide. Our nanopillars are, comparatively, much larger structures but the separation between them can be engineered with much greater ease down to around $10 \mathrm{~nm}$. It's easier to control that gap size than the size of the tip of a needle.

\section{What have you trapped?}

We've used our tweezers to trap 200-nmdiameter polystyrene beads immersed in glycerol, as a way of demonstrating the basic operation and efficiency of our optical traps. But the idea is to eventually trap more complicated biological objects.

\section{How strong are your traps?}

Optical tweezing is quantified in terms of 'trapping quality', which gives a measure of how effectively a particle can be pinned at a given laser power. It is basically the ratio of the trapping force generated to the power of the laser used to achieve the trapping (with some extra coefficients folded in). In our experiments, we measure the trapping forces by moving the laser beam across the nanostructured substrate, moving the trap from one nanopillar to the next and mapping the motion of the beads as they follow the beam. We measure their terminal velocity as they become detached from the moving trap and relate this to the trapping force. The larger the force applied, the higher the velocity.

The results show that nanometric tweezers offer a pretty huge improvement in trapping quality compared with conventional optical tweezers. The trapping quality is 14 for $6-\mu \mathrm{m}$ beads and about 0.1 for 200 -nm beads. For a given power, nanotweezers offer an improvement by about a factor of 10-20 in the positional control of the trapped object.

How do you know the trapped objects are being held in place by optical forces and not mechanical ones?

It's possible that there are some mechanical contributions to the particle confinement. But it comes down to the escape-velocity measurements when we move the trap from one nanopillar pair to the next. You cannot generate a mechanical force that allows particles to follow the light beam with the speeds that we have measured - about 20 times faster than observed in ordinary far-field tweezing. That's why we believe optical forces are responsible.

\section{Where next?}

We are working on optimizing our nanopillar structures. We need to show that we can trap and move a single molecule reliably and then measure its properties reliably. It should be pretty straightforward to perform single-molecule analysis using our trapping scheme - a particle could be trapped and then nonlinear processes, such as surface-enhanced Raman scattering or other effects, could be used to probe it. Alternatively, you could perform high-throughput analysis and probe many particles at once using a large number of nanotraps that segregate different objects. The future of nanotweezing probably lies in combining its trapping capabilities with other sensing techniques. We're working with biologists to see where it could go.

Interview by Amber Jenkins.

Grigorenko and his colleagues have a Letter on nanometric optical tweezers on page 365 of this issue. 